\title{
El metilfenidato reduce la apatía en pacientes con Alzheimer
}

\author{
Methylphenidate reduces apathy in patients with Alzheimer's disease
}

\section{Comentado de:}

Padala PR, et al. Am J Psychiatry 2018; 175(2):159-168. PMID $28945120^{1}$

\section{Objetivo}

Evaluar la efectividad del metilfenidato en el tratamiento de la apatía enveteranos con diagnóstico de Alzheimer leve en un periodo de 12 semanas.

\section{Diseño y lugar}

Ensayo clínico aleatorizado, doble ciego controlado con placebo, llevado a cabo en un centro médico de Veteranos de Guerra de los EE.UU.

\section{Población}

Participaron pacientes con diagnóstico de Alzheimer y un minimental test mayor o igual a 18, una escala de apatía clínica mayor a 40 , que tuviesen un cuidador disponible y, en caso de consumo de inhibidores de la acetilcolinesterasa o antidepresivos, un tiempo de administración del fármaco mayor a 4 meses y 2 meses, respectivamente.

\section{Intervención}

Los pacientes fueron asignados al azar, por aleatorización en bloques, a dos grupos: metilfenidato o placebo, y fueron seguidos por 12 semanas. Los participantes, el equipo de estudio y los evaluadores de resultado estaban enmascarados para la asignación de tratamiento. La medicación del estudio fue contada y dispensada por la farmacia de investigación. Todos los participantes comenzaron con $5 \mathrm{mg}$ de metilfenidato (o placebo similar) dos veces al día, y fue titulado a $10 \mathrm{mg}$ a las 2 semanas.

\section{Medición de Resultados Principales}

La medida de resultado primario fue la puntuación en la escala clínica de apatía (AES-C). Esta escala va de 18 a 72, con un valor de más de 40 para considerar apatía en demencia. Se consideró una mejora significativa a partir de 3 puntos de cambio en la escala.

Las medidas de resultado secundarias fueron evaluadas a partir de las siguientes escalas: Mini-Mental convencional (MMSE) y modificado (3MS), actividades instrumentales de la vida diaria (AVDI), actividades de la vida diaria (AVD), escala de Zarit Burden para carga del cuidador, escalas de evaluación global general (CGI-S y CGI-I ), y la escala de Cornell para la depresión en pacientes con demencia. Además se reportaron los eventos adversos. Los resultados se analizaron por protocolo.

\section{Resultados Principales}

Se incluyeron 60 participantes; 30 en el grupo MFD y 30 en el grupo placebo. Todos fueron hombres (edad promedio 77 años, desvío estándar $=8$ ). La pérdida fue simétrica en ambos grupos (se perdió un participante por cada rama). Se reportaron 22 efectos adversos, de los cuales 6 fueron graves con hospitalizaciones (5 con metilfenidato y 1 con placebo). Los resultados principales se resumen en la Tabla 1 y la Tabla 2.

Tabla 1. Cambios en las escalas de apatía y de cognición en el seguimiento a 4 semanas

\begin{tabular}{|l|c|c|c|}
\hline Desenlaces & $\begin{array}{c}\text { Metilfenidato, } \\
\text { diferencia (IC } \\
95 \%)\end{array}$ & $\begin{array}{c}\text { Placebo, } \\
\text { diferencia (IC } \\
95 \%)\end{array}$ & p-valor \\
\hline $\begin{array}{l}\text { Escala de } \\
\text { apatía (AES- } \\
\text { C) }\end{array}$ & $-9,7(-12.3$ a 7,1$)$ & $-4,4(-7$ a 1,8$)$ & 0,006 \\
\hline $\begin{array}{l}\text { Mini Mental } \\
\text { test conven- } \\
\text { cional }\end{array}$ & 1 (-0,1 a 2) & $1(0,0$ a 2,1$)$ & 0,921 \\
\hline
\end{tabular}

Tabla 2. Cambios en las escalas de apatía y de cognición en el seguimiento a 12 semanas

\begin{tabular}{|l|c|c|c|}
\hline Desenlaces & $\begin{array}{c}\text { Metilfenidato, } \\
\text { diferencia (IC } \\
95 \%)\end{array}$ & $\begin{array}{c}\text { Placebo, } \\
\text { diferencia (IC } \\
95 \%)\end{array}$ & p-valor \\
\hline $\begin{array}{l}\text { Escala de } \\
\text { apatía (AES- } \\
\text { C) }\end{array}$ & $\begin{array}{c}-14,1(-16,8 \mathrm{a} \\
-11,6)\end{array}$ & $-4,2(-6,8 \mathrm{a}-1,7)$ & $<0,001$ \\
\hline $\begin{array}{l}\text { Mini Mental } \\
\text { test conven- } \\
\text { cional }\end{array}$ & $2,2(1,2$ a 3,2) & $-0,4(-0,4$ a 1,6) & 0,001 \\
\hline
\end{tabular}

\section{Conclusiones}

En pacientes con apatía y demencia leve, el metilfenidato se asoció con una mejora significativamente mayor en comparación con el placebo después de 4 semanas de tratamiento, que continuó creciendo con el seguimiento a las 8 y 12 semanas. Además se mostraron mejoras significativamente mayores que en el grupo placebo en otros desenlaces como la cognición, el estado funcional, la carga del cuidador y la depresión, aunque estos efectos no se vieron hasta las 12 semanas.

Fuente de financiamiento/Conflicto de interés de los autores: No referidos

\section{Comentario}

La apatía se encontró en el $58 \%$ de los casos de pacientes con demencia ${ }^{2}$. En países como España, algunas guías describen como alternativa al metilfenidato para el tratamiento de este síntoma ${ }^{3}$, sin embargo en nuestra práctica diaria, este 
fármaco no se utiliza para tal fin.

Este estudio mostró efectividad del metilfenidato para disminuir la apatía en pacientes con demencia tipo Alzheimer leve, y para mejora la cognición, del mismo modo que los inhibidores de acetilcolinesterasa. Sus resultados son consistentes con los de una revisión sistemática Cochrane publicada casi en simultáneo, que incluyó 3 ensayos clínicos aleatorizados (incluyendo el resumido en este artículo) que compararon metilfenidato con placebo para el tratamiento de la apatía en pacientes con Alzheimer. Sin embargo, debido a la inconsistencia entre los estudios y a la imprecisión de la medida sumaria, se consideró que el efecto beneficioso sobre la apatía (Diferencia media -4,99; IC $95 \%-9,55$ a - - 4,43) cuenta con baja calidad de evidencia ${ }^{4}$.

Una de las limitaciones para la interpretación de los resultados del estudio (incluso, de la revisión sistemática mencionada) es que sólo informa las diferencias observadas mediante un promedio de cambio en la escala, y no como una variable dicotómica que pudiese expresar en cuántos pacientes es observó un cambio clínicamente relevante en la apatía y en cuantos esta no se modificó. Por otro lado, la población fue masculina en su totalidad, por lo que no podemos estar seguros si los resultados son extrapolables a la población femenina.

Los efectos adversos fueron bastante prevalentes para el número de pacientes evaluados; sin embargo, en la revisión sistematica este desenlace tuvo una medida sumaria inconcluyente (Riesgo Relativo 1,28; IC 95\% 0,67 a 2,42) y de baja calidad de evidencia, motivo por el cual el riesgo del uso de metilfenidato continúa siendo desconocido, sobre todo en poblaciones ancianas con comorbilidades ${ }^{4}$. El mantenimiento del efecto y el pronóstico de los pacientes tratados con esta droga son interrogantes que todavía no tienen respuestas.

\section{Conclusiones del comentador}

En un grupo seleccionado de pacientes con demencia leve, el metilfenidato podría ser una opción con cierta evidencia para los síntomas de apatía, aunque su perfil de seguridad es dudoso, al igual que la duración del efecto buscado.

María Florencia Herrera Bornes [ Servicio de Medicina Familiar y Comunitaria, Hospital Italiano de Buenos Aires florencia.herrera@Hospitalitalino.org.ar ]

Herrera Bornes, MF. El metilfenidato reduce la apatía en pacientes con Alzheimer. Evid Actual Pract Ambul. 2019;22(4):e001090. Comentado de: Padala PR, et al. Methylphenidate for Apathy in Community-Dwelling Older Veterans With Mild Alzheimer's Disease: A Double-Blind, Randomized, PlaceboControlled Trial. Am J Psychiatry 2018; 175(2):159-168. PMID 28945120

\section{Referencias}

1. Padala PR, Padala KP, Lensing SY, Ramirez D, Monga V, Bopp MM, et al. Methylphenidate for Apathy in Community-Dwelling Older Veterans With Mild Alzheimer's Disease: A Double-Blind, Randomized, Placebo-Controlled Trial. Am J Psychiatry. 2018;175(2):159-168. Available from: 10.1176/appi.ajp.2017.17030316

2. Baldin-Storti L, Teles-Quintino D, Michelato-Silva N, Kusumota L, Marques S. Síntomas neuropsiquiátricos de adultos mayores con enfermedad de Alzheimer y el desgaste de los cuidadores familiares. Rev Latino-Am Enfermagem. 2016;24:e2751. cited. Available from: 10.1590/1518-8345.0580. 2751.

3. Arrieta-Anton E, Gomariz-Garcia JJ, Rodriguez-Fernandez RM. Know Alzheimer. Respuestas concretas a dudas reales. Manual de consulta para Médicos de Atención Primaria. Martinez-Lage P, editor. Barcelona: Profarmaco.2 Formación Continuada; 2014. Available from: www.sefac.org/sites/ default/files/sefac2010/private/documentos_sefac/documentos/KA_Manual_AP_0.pdf.

4. Ruthirakuhan MT, Herrmann N, Abraham EH, Chan S, Lanctôt $\bar{K}$. Pharmacological interventions for apathy in Alzheimer's disease. Cochrane Database Syst Rev. 2018;5:CD012197. Available from: 10.1002/14651858.CD012197.pub2. 